\title{
Dímero D como predictor de gravedad en el paciente con síndrome de insuficiencia respiratoria aguda por SARS-CoV-2
}

\author{
Daniel A. Montalvo-López ${ }^{1 *}$, Hermes M. Cortés-Meza ${ }^{1}$, Fabiola Vivanco-Salazar², Alfredo Tanaka-Chávez ${ }^{1}$ \\ y Francisco G. Reyes-Rodríguez ${ }^{1}$ \\ ${ }^{1}$ Servicio de Urgencias Adultos; ${ }^{2}$ Servicio de Neuroanestesiología. Hospital Juárez de México, Secretaría de Salud, Ciudad de México, México
}

\begin{abstract}
Resumen
Introducción: La reciente pandemia por el coronavirus 2 del síndrome respiratorio agudo grave (SARS CoV-2) ha planteado nuevos retos médicos en el tratamiento de todos aquellos pacientes con manifestaciones severas. Al respecto, existe poca evidencia en relación con la coagulopatía e interpretación de los niveles de dímero $D$, pese a lo cual se ha observado de forma repetida que los niveles elevados de dímero $D$ se correlacionan con la severidad de la respuesta inflamatoria sistémica y a su vez con la gravedad del paciente, siendo un predictor útil para guiar el tratamiento de estos pacientes. Material y método: Estudio observacional, retrospectivo transversal, que se realizó en el Servicio de Urgencias Adultos del Hospital Juárez de México del 1 abril al 30 de julio del 2020. Los criterios de inclusión fueron: ser mayor de 18 años, saturación de oxígeno al ingreso $<80 \%$, frecuencia respiratoria al ingreso $>20$, insuficiencia respiratoria tipo 1 (definida como: $\mathrm{pO}_{2}<55 \mathrm{mmHg}$ ), incremento del dímero $D \geq 3$ veces el valor de referencia, es decir, valores iguales o mayores a 1,000 ng/dl y ser positivo a enfermedad por coronavirus-2019 (definido como una r-RT-PCR positiva). Se excluyeron pacientes con antecedente de infarto agudo al miocardio, evento vascular cerebral o trombosis venosa profunda en los últimos seis meses, así como pacientes que llegaran al servicio de urgencias en paro cardiaco o que fallecieron durante las primeras 24 horas de atención. Resultados: Del total de pacientes registrados, fallecieron 86 (84\%). La mortalidad en pacientes con dímero $D$ superior a 1,500 fue del 97.3\% ( $p=0.003)$. Se observó que en los pacientes con dímero $D>1,500$ el riesgo de morir era 11 veces mayor que en los pacientes con dímero $D$ menor, con una odds ratio de 11.33 (intervalo de confianza del 95\%: 1.43-89.65). Conclusiones: Los pacientes infectados por el SARS-CoV-2 que muestran manifestaciones severas presentan una elevación anormal del dímero $D$. Se ha demostrado que las cifras de dímero $D$ se correlacionan estrechamente con la gravedad del paciente, por lo cual puede utilizarse como marcador pronóstico.
\end{abstract}

Palabras clave: SARS CoV-2. Anticoagulantes. Dímero D. Mortalidad. Pronóstico.

\section{D-dimer as a predictor of severity in the patient with ARDS due to SARS-CoV-2}

\section{Abstract}

Introduction: The recent SARS CoV-2 pandemic has posed new medical challenges in the management of all those patients with severe manifestations. In this regard, there is little evidence regarding coagulopathy and interpretation of D-dimer levels, however, it has been repeatedly observed that elevated D-dimer levels correlate with the severity of the systemic inflammatory

\section{Correspondencia:}

*Daniel A. Montalvo-López

E-mail: danomontalvo@ hotmail.com
Disponible en internet: 13-09-2021

Rev Educ Investig Emer. 2021;3(3):120-126 www.medicinadeemergencias.com bajo la licencia CC BY-NC-ND (http://creativecommons.org/licenses/by-nc-nd/4.0/). 
response and in turn with the severity of the patient, being a useful predictor to guide the treatment of these patients. Methods: Observational, retrospective, cross-sectional, retrospective study was conducted in the Adult Emergency Department of the Hospital Juarez de Mexico from April 1 to July 30, 2020. Inclusion criteria were patients older than 18 years, oxygen saturation on admission $<80 \%$, respiratory rate on admission $>20$, type 1 respiratory failure (defined as: p02 $<55 \mathrm{mmHg}$ ), D-dimer increase $\geq 3$ times the reference value, i.e. values equal to or greater than $1000 \mathrm{ng} / \mathrm{dl}$, positive COVID-19 defined as a positive $r$-RT-PCR. Patients with a history of acute myocardial infarction, cerebral vascular event or deep vein thrombosis in the last 6 months were excluded, as well as patients who arrived at the emergency department in cardiac arrest or who died during the first 24 hours of care. Results: Of the total number of patients registered, 86 (84\%) died. Mortality in patients with $D$-dimer greater than 1500 was $97.3 \%(p=0.003)$. It was observed that in patients with $D$-dimer $>1500$ the risk of death was 11 times higher than in patients with lower D-dimer, with an OR of 11.33 (95\% Cl 1.43-89.65). Conclusions: Patients infected with SARS- CoV-2 virus showing severe manifestations have an abnormal elevation of D-Dimer. It has been shown that D-Dimer figures correlate closely with patient severity, so it can be used as a prognostic marker.

Key words: SARS CoV-2. Anticoagulants. D-dimer. Mortality. Prognosis.

\section{Introducción}

En la época actual nos enfrentamos a una nueva enfermedad denominada COVID 19, acrónimo asignado al coronavirus 19 , agente causal de un síndrome respiratorio agudo que en casos graves puede determinar dificultad respiratoria y evolucionar, de forma rápida, hacia insuficiencia respiratoria aguda, fracaso multiorgánico $\mathrm{y}$, finalmente, a la muerte. Alrededor de 70 días después de haberse informado los primeros casos de esta enfermedad, la Organización Mundial de la Salud declaró la existencia de una pandemia relacionada con este virus emergente, cuya letalidad es del $4.38 \% \%^{1,2}$.

La enfermedad grave suele comenzar aproximadamente una semana después de la aparición de los síntomas iniciales. La disnea es el síntoma más común de enfermedad grave y suele ir acompañada de hipoxemia. En muchos pacientes con COVID-19 grave se desarrolla insuficiencia respiratoria progresiva poco después de la aparición de disnea e hipoxemia. Se estima que alrededor del $42 \%$ de los pacientes que presentan neumonía desarrollaran síndrome de dificultad respiratoria aguda (SDRA). Estos pacientes comúnmente cumplen los criterios para SDRA, que se define como la aparición aguda de infiltrados bilaterales, hipoxemia grave y edema pulmonar que no se explica completamente por insuficiencia cardiaca o sobrecarga de líquidos ${ }^{3}$. De acuerdo con reportes iniciales, la mortalidad por SDRA asociado a SARS-CoV-2 oscila entre el 26 y el $61.5 \%$, siendo incluso más alta para aquellos que requieren ventilación mecánica ${ }^{4}$.

Uno de los factores clave para la invasión celular lo representa la enzima convertidora de angiotensina 2 (ECA2), la cual ha sido reconocida como un receptor necesario para el ingreso del SARS-CoV-2 a las células hospederas; este receptor se expresa ampliamente en tejidos cardiopulmonares, pero también en algunas células hematopoyéticas, incluidos monocitos y macrófagos $^{5}$. En condiciones normales, la ECA2 actúa sobre la angiotensina, para formar angiotensina 1-7, la cual se encarga de favorecer la síntesis de óxido nítrico, compuesto que produce vasodilatación y suprime la agregación plaquetaria. La unión del SARS-CoV-2 a la ECA2 agrava el daño celular, aumentando la expresión del factor tisular y regulando hacia la baja el sistema de la proteína C. En esta situación, se producen fácilmente coagulopatía y eventos trombóticos ${ }^{6}$.

Se ha identificado que la nueva infección por COVID-19 provoca un estado procoagulante, con trombosis a nivel venoso y arterial, por medio de la activación del factor von Willebrand almacenado en el cuerpo de Weibel Palade, el cual se libera a la circulación, promoviendo la formación de coágulos, bajo la activación de la interleucina (IL) $1 \beta$ e IL-6; induciendo hiperfibrinogenemia y trombocitosis, la cual se acompaña de niveles elevados de dímero $\mathrm{D}$, con trombocitopenia leve $^{3}$. La elevación del dímero $D$ se explica por la regulación positiva de la fibrinólisis local en los alvéolos por el activador del plasminógeno de tipo urocinasa liberado por los macrófagos alveolares. Además, la infección directa de las células endoteliales por el virus conduce a una liberación masiva de activadores del plasminógeno'.

Con un aumento en la gravedad de la enfermedad, hay un cambio procoagulante con la aceleración de la formación de fibrina producida por niveles aumentados de fibrinógeno y plaquetas activadas. La fibrinólisis suprimida por la liberación del inhibidor del activador de plasminógeno 1 acelera la formación de coágulos en los capilares pulmonares ${ }^{6}$. 
La hipótesis principal fundamenta que la inflamación sistémica favorece la activación de la cascada de coagulación y del sistema fagocítico mononuclear, sistemas que exacerban la inflamación generalizada en todos los aparatos y sistemas y promueven, simultáneamente, la cascada de coagulación; ambos efectos generan un circulo vicioso en el cual diversas proteínas del sistema reticuloendotelial generan más inflamación, lesión del endotelio y mayor generación de trombos, ante lo cual se ha visualizado como un probable objetivo hacia el cual dirigir el tratamiento?.

Existe un interés particular en la coagulopatía en pacientes con COVID-19, ya que los parámetros de coagulación anormales, las elevaciones más consistentes en el dímero $D$ y los productos de degradación de la fibrina (FDP) están asociados con la gravedad de la enfermedad. Un dímero $D$ elevado, la anomalía de coagulación más común en COVID-19 (que se encuentra en hasta el $45 \%$ de los pacientes) es un factor de riesgo independiente de muerte; se ha observado que pacientes con dímero $D$ mayores de $1.000 \mathrm{ng} / \mathrm{ml}$ tienen casi 20 veces más probabilidades de morir a causa de la infección que los pacientes con valores de dímero $D$ más bajos ${ }^{8,9}$. Por el contrario, la mayoría de los pacientes con COVID-19 tienen un tiempo de protrombina normal o levemente prolongado y un tiempo de tromboplastina parcial activada normal o acortado en la presentación, sin que estos laboratorios se asocien de manera confiable con la gravedad de la enfermedad. Tanto la monitorización inicial como longitudinal de los parámetros de coagulación pueden predecir la gravedad de la enfermedad. Se ha observado que niveles elevados de dímero D y de FDP, así como niveles disminuidos de fibrinógeno y antitrombina III, se asocian con elevación de la mortalidad ${ }^{10}$, por lo cual se recomienda monitorizar los niveles de dímero $D$ al ingreso y cada 24-48 h como herramienta en la evaluación del pronóstico y de la progresión de la enfermedad ${ }^{11}$.

El hecho de que una coagulopatía esté presente en estos pacientes ha promovido que se planteen estrategias antitrombóticas, sobre todo en los pacientes que ingresan en la unidad de cuidados intensivos (UCI) y/o muestran daño orgánico o episodios isquémicos; esto ha llevado a los proveedores de primera línea a considerar el uso empírico de anticoagulación terapéutica para pacientes hospitalizados, incluso en ausencia de trombosis documentada o clínicamente sospechada ${ }^{12}$. Si bien aún no se ha establecido la mejor estrategia antitrombótica, parece que las heparinas de bajo peso molecular (HBPM), a dosis profilácticas o intermedias, deberían indicarse en estos pacientes, tras su ingreso en la $\mathrm{UCl}$ o cuando los valores de dímero $\mathrm{D}$ sean cuatro veces superiores a los normales ${ }^{8,9}$.

En varios reportes, desde el inicio de esta enfermedad, se habla del efecto benéfico de la heparina en pacientes con cifras elevadas de dímero $D o$ en quienes tienen manifestaciones francas de trombosis, asociándose con una disminución del número de muertes en un lapso de 28 días (40 vs. $64.2 \% ; p=0.029)^{10}$. Tang, et al. describieron que la terapia anticoagulante principalmente con HBPM parecía estar asociada con un mejor pronóstico en 449 pacientes con COVID-19 grave que cumplían los criterios de coagulopatía inducida por sepsis $\geq 4$ o con niveles marcadamente elevados de dímero $D$ (más de seis veces en el límite superior) de lo normal ${ }^{11}$. A este respecto, las recomendaciones actuales de expertos, incluidas las pautas provisionales de la Sociedad Internacional de Trombosis y Hemostasia (ISTH) y el Colegio Americano de Cardiología (ACC), recomiendan el uso de dosis profilácticas de HBPM o heparina no fraccionada en todos los pacientes con COVID-19 que requieran ingreso hospitalario ${ }^{12}$. Para pacientes críticamente enfermos con COVID-19 confirmado o altamente probable, la ISTH sugiere dosis mayores de profilaxis de tromboembolia venosa (p. ej., enoxaparina $40 \mathrm{mg}$ subcutánea dos veces al día, enoxaparina $0.5 \mathrm{mg} / \mathrm{kg}$ subcutánea dos veces al día, heparina 7,500 unidades subcutáneas tres veces al día o infusión de heparina de baja intensidad ${ }^{13,14}$.

La terapia con heparina tiene varias ventajas: a) representa, en tiempos de una pandemia, una terapia anticoagulante fácilmente disponible, dadas las preocupaciones iniciales con respecto a la escasez de medicamentos; $b$ ) se han informado efectos antiinflamatorios incrementales y puede mitigar las tormentas de citocinas en pacientes con COVID-19 grave; c) los modelos experimentales informaron de un posible papel antiviral de la heparina aún por confirmar en la práctica clínica y en el contexto de la infección por SARS-CoV-2, y finalmente d) actualmente no hay evidencia de ensayos clínicos aleatorizados de que cualquier terapia potencial mejore los resultados en pacientes con COVID-19 sospechada o confirmada ${ }^{11}$.

El objetivo del presente estudio fue determinar el papel del dímero $D$ como predictor de mortalidad en pacientes con SDRA infectados por SARS-CoV-2 que ingresaron en la unidad de urgencias del Hospital Juárez de México en el periodo comprendido del 1 abril al 30 de julio del 2020, lo anterior con la finalidad de estipular la población que más se beneficiaría del uso temprano de HBPM. 


\section{Material y métodos}

Se trata de un estudio observacional, retrospectivo, transversal, que se realizó en el servicio de urgencias adultos del Hospital Juárez de México del 1 abril al 30 de julio del 2020. Los criterios de inclusión fueron pacientes mayores de 18 años, saturación de oxígeno al ingreso $<80 \%$, frecuencia respiratoria al ingreso $>20$, insuficiencia respiratoria tipo 1 (definida como $\mathrm{PaO} 2<55 \mathrm{mmHg}$ ), incremento del dímero $\mathrm{D} \geq 3$ veces el valor de referencia (es decir, valores iguales o mayores a $1,000 \mathrm{ng} / \mathrm{dl}$ ), COVID-19 positiva definida como una RT-PCR positiva. Se excluyeron pacientes con antecedente de infarto agudo al miocardio, evento vascular cerebral o trombosis venosa profunda en los últimos seis meses, así como pacientes que llegaran al servicio de urgencias en paro cardiaco o que fallecieron durante las primeras 24 horas de atención. El análisis estadístico se llevo a cabo en SPSS versión 23.0. Las variables cuantitativas se describieron como medias y desviación estándar; las cualitativas se describieron con porcentajes. Se realizó Chi cuadrada para las variables con distribución distinta a la normal y t de Student para variables con distribución normal. Se tomó un valor de $p<0.005$ para significancia estadística. Se calcularon odds ratios (OR) sin ajustar para intubación y muerte.

El protocolo fue aprobado por los comités de investigación y/o ética en investigación hospitalarios.

\section{Resultados}

Se recolectaron datos de 102 casos consecutivos que ingresaron al servicio de urgencias adultos del hospital y cumplieron los criterios de inclusión.

De los 102 pacientes el $32.4 \%(n=33)$ eran mujeres $y$ el $67.4 \%(n=69)$ eran hombres; con un media de edad de $58.13 \pm 12.72$ años, una edad máxima de 79 años y mínima de 33 años.

En cuanto a la presencia de comorbilidades reportadas: el $56.9 \%(n=58)$ de los pacientes tuvieron diagnóstico de diabetes tipo 2; el $48 \%(\mathrm{n}=49)$ de hipertensión arterial sistémica (HAS), el $51 \%(n=52)$ enfermedad pulmonar obstructiva crónica y el $50 \%$ de tabaquismo (Tabla 1).

En cuanto a los signos vitales al ingreso, se registró una media de tensión arterial sistólica de $138.84 \pm$ $18.30 \mathrm{mmHg}$, con una presión arterial diastólica promedio de $81.44 \pm 8.7 \mathrm{mmHg}$. La frecuencia cardiaca al ingreso fue de media $100 \pm 8.56 \mathrm{lpm}$, el número de respiraciones por minuto fue de $32 \pm 3.9$ respiraciones por minuto; con
Tabla 1. Características basales de la población. Comorbilidades más frecuentes del total de la población incluida en el estudio

\begin{tabular}{|l|c|}
\hline \multicolumn{2}{|c|}{ Características basales de la población } \\
\hline Edad, media & $58.13 \pm 12.72$ \\
\hline Hombres n (\%) & $71(69.6)$ \\
\hline Tabaquismo n (\%) & $51(50)$ \\
\hline DM2 n (\%) & $58(56.8)$ \\
\hline HAS n (\%) & $49(48)$ \\
\hline EPOC n (\%) & $52(50.9)$ \\
\hline
\end{tabular}

DM2: diabetes mellitus tipo 2; HAS: hipertensión arterial sistémica; EPOC: enfermedad pulmonar obstructiva crónica.

Tabla 2. Registro de las constantes vitales de los pacientes a su ingreso a la Unidad de Urgencias Respiratorias de Adultos; se presenta la media y la desviación estándar de cada valor

\begin{tabular}{|lc|}
\hline \multicolumn{2}{|c|}{ Signos vitales al ingreso } \\
\hline Tensión arterial sistólica, $\mathrm{mmHg}$ & $138.84 \pm 18.30$ \\
\hline Tensión arterial diastólica, $\mathrm{mmHg}$ & $81.44 \pm 8.7$ \\
\hline Frecuencia cardiaca, Ipm & $100 \pm 8.56$ \\
\hline Frecuencia respiratoria, rpm & $32 \pm 3.9$ \\
\hline Pulsioximetría, \% & $76.74 \pm 8.56$ \\
\hline Temperatura, ${ }^{\circ} \mathrm{C}$ & $37.25 \pm 0.64$ \\
\hline
\end{tabular}

una pulsioximetría promedio de $76.74 \pm 8.56$ y una temperatura corporal promedio de $37.25 \pm 0.64 \stackrel{\circ}{ } \mathrm{C}$ (Tabla 2).

Del total de pacientes registrados, el $37.2 \%(n=38)$ tuvo un valor de dímero $\mathrm{D} \geq 1,500 \mathrm{ng} / \mathrm{dl}$, con un valor máximo de 76,800 ng/dl (Fig. 1).

En cuanto al manejo de la vía aérea, del total de los pacientes incluidos, el $82.4 \%(n=84)$ ameritó intubación orotraqueal (Tabla 3).

Del total de pacientes $(n=102)$, el $62.7 \%$ contaba con cifras de dímero D menores a 1,500 ng/dl comparado con el $37.2 \%$ que contaba con cifras superiores a $1,500 \mathrm{ng} / \mathrm{dl}$. En relación con el riesgo de intubación, se observó que los pacientes con dímero $D$ mayor o igual a 1,500 tenían hasta 13 veces el riesgo de requerir ventilación mecánica invasiva comparada con aquellos con valores menores de dímero D (OR: 13.38; intervalo de confianza del 95\% [IC 95\%]: 1.7-105.2; $p=0.001$ ) (Tabla 4). 


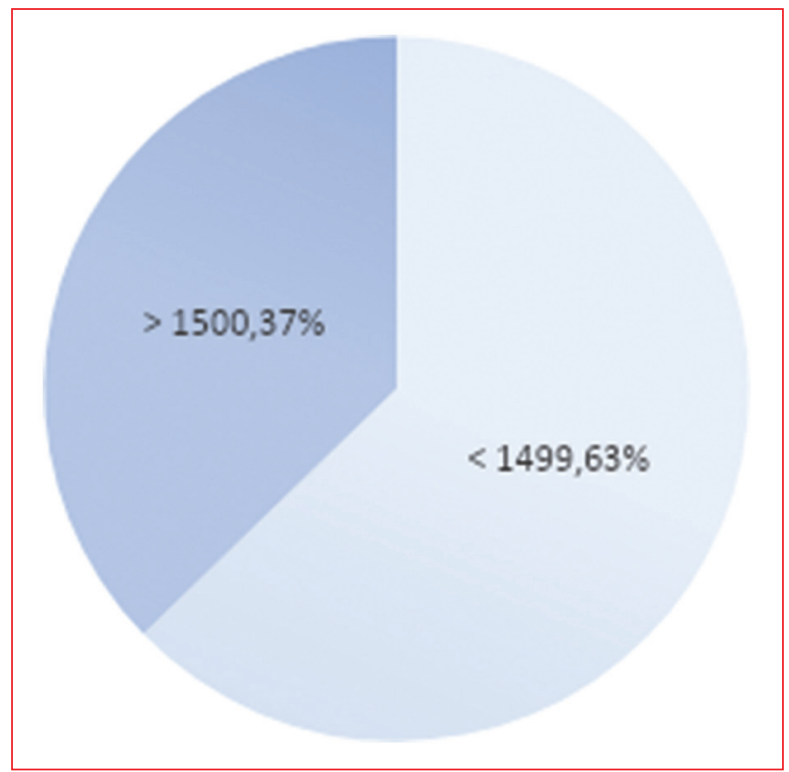

Figura 1. Gráfica que representa el porcentaje de población con cifras de dímero $D$ menores a 1,500 $\mathrm{ng} / \mathrm{dl}$ y aquellos que contaban con cifras mayores a $1,500 \mathrm{ng} / \mathrm{dl}$

Tabla 3. Pacientes intubados. Registro del total de pacientes participantes, de los cuales el $82.4 \%$ ameritó manejo avanzado de la vía aérea en contraposición con el $17.6 \%$ que no lo requirió

\begin{tabular}{|l|c|c|}
\hline \multicolumn{3}{|c|}{ Pacientes intubados } \\
\hline & $\mathbf{n}$ & $\%$ \\
\hline Sí & 84 & 82.4 \\
\hline No & 18 & 17.6 \\
\hline Total & 102 & 100 \\
\hline
\end{tabular}

Del total de pacientes registrados, fallecieron 86 (84\%). La mortalidad en pacientes con dímero $D$ superior a 1,500 fue del $97.3 \%$, mientras que en los pacientes con dímero $D$ menor de 1,499 fue del 76\% ( $p=0.003$ ). Se observó que los pacientes con dímero $D \geq 1,500$ el riesgo de morir era 11 veces mayor que en los pacientes con dímero D menor, con una OR de 11.33 (IC 95\%: 1.43-89.65). De los pacientes intubados con dímero $D$ mayor a 1,500 hubo una mortalidad del 100\% (Tabla 5).

Del total de los expedientes registrados se observó que los pacientes con dímero $D<1,499$ tuvieron una menor frecuencia de síndrome de insuficiencia respiratoria aguda (SIRA) severo en comparación de los pacientes con dímero $D \geq 1,500 \mathrm{ng} / \mathrm{dl}$ (56 vs. $76 \%$, respectivamente; $p=0.033$ ) (Tabla 6 ).
Tabla 4. Correlación del valor de Dímero $D$ y manejo avanzado de la vía aérea. Obsérvese que del total de pacientes ( $n=102$ ), el $62.7 \%$ contaba con cifras de dímero $\mathrm{D}$ menores a 1,500 ng/dl, comparado con el $37.2 \%$ que contaba con cifras superiores a $1,500 \mathrm{ng} / \mathrm{dl}$. En relación con el riesgo de intubación, se observó que los pacientes con dímero $D$ mayor o igual a 1,500 tenían hasta 13 veces el riesgo de requerir ventilación mecánica invasiva comparada con aquellos con valores menores de dímero D (OR: 13.38; IC 95\%: 1.7-105.2; $p=0.001$ )

\begin{tabular}{|l|c|c|c|}
\hline \multicolumn{3}{|c|}{ Tabla de contingencia para intubación y valores de dímero D } \\
\hline & \multicolumn{2}{|c|}{ Intubación } & \multirow{2}{*}{ Total } \\
\cline { 2 - 3 } & Sí & No & \\
\hline $\begin{array}{c}\text { Dímero D } \\
\text { (ng/dl) } \\
<1,499 \\
n\end{array}$ \\
$\begin{array}{c}\mathrm{n} 1,500 \\
\mathrm{n}\end{array}$ \\
\hline \begin{tabular}{c} 
Total \\
\hline
\end{tabular} & $47(73 \%)$ & $17(27 \%)$ & 64 \\
\hline
\end{tabular}

OR: odds ratio; IC 95\%: intervalo de confianza del $95 \%$

Tabla 5. Correlación del valor de dímero D y mortalidad. Obsérvese que del total de pacientes registrados, fallecieron 86 (84\%). La mortalidad en pacientes con dímero $\mathrm{D}$ superior a 1,500 fue del $97.3 \%$, mientras que en los pacientes con dímero $\mathrm{D}$ menor de 1,499 fue del $76 \%$ $(p=0.003)$. Se observó que los pacientes con dímero $D>1,500$ el riesgo de morir era 11 veces mayor que en los pacientes con dímero $\mathrm{D}$ menor, con una OR de 11.33 (IC 95\%: 1.43-89.65). De los pacientes intubados con dímero $D$ mayor a 1,500 hubo una mortalidad del $100 \%$

\begin{tabular}{|l|c|c|c|}
\hline \multicolumn{3}{|c|}{ Tabla de contingencia para mortalidad y valores de dímero D } \\
\hline & \multicolumn{2}{|c|}{ Muerte } & \multirow{2}{*}{ Total } \\
\cline { 2 - 3 } & Sí & No & \\
\hline $\begin{array}{c}\text { Dímero D } \\
\text { (ng/dl) } \\
<1499 \\
n \\
\begin{array}{c}n \\
n\end{array}\end{array}$ & $49(76 \%)$ & $15(23 \%)$ & 64 \\
\hline \begin{tabular}{c} 
Total \\
\hline
\end{tabular} & $37(97.3 \%)$ & $1(5.6 \%)$ & 38 \\
\hline
\end{tabular}

OR: odds ratio; IC 95\%: intervalo de confianza del $95 \%$.

\section{Discusión}

El dímero $D$ es un subproducto generado cuando la plasmina degrada los coágulos de fibrina; y constituye 
Tabla 6. Correlación del valor de dímero D y SIRA. Del total de los expedientes registrados se observó que los pacientes con dímero $D<1,499$ tuvieron una menor frecuencia de SIRA severo en comparación de los pacientes con dímero $\mathrm{D}>1,500 \mathrm{ng} / \mathrm{dl}$ ( $56 \mathrm{vs}$. $76 \%$, respectivamente; $p=0.033$ )

\begin{tabular}{|l|c|c|c|}
\hline \multirow{2}{*}{ Dímero D y SIRA } \\
\hline & \multicolumn{2}{|c|}{ SIRA } & \multirow{2}{*}{ Total } \\
\cline { 2 - 3 } & Severo & Moderado & \\
\hline $\begin{array}{c}\text { Dímero D } \\
\text { (ng/d) } \\
<1499 \\
n \\
\geq 1500 \\
n\end{array}$ & $36(56 \%)$ & $28(43 \%)$ & \\
\hline Total & $29(76 \%)$ & $9(24 \%)$ & 38 \\
\hline
\end{tabular}

SIRA: síndrome de insuficiencia respiratoria aguda.

un marcador útil de la actividad trombótica. Debido a su importancia pronóstica, se ha convertido en un marcador de laboratorio útil para valorar la severidad de la enfermedad en individuos hospitalizados con COVID-19.

Se ha demostrado que en los pacientes diagnosticados con neumonía atípica secundaria a SARS-CoV-2, la elevación del dímero $\mathrm{D}$ a su ingreso hospitalario es común y se vincula con una presentación más severa de la enfermedad, así como a elevación de la mortalidad hospitalaria.

Pese a lo anterior, el dímero $D$ no se ha utilizado como biomarcador de la neumonía viral, aunque se ha registrado su elevación como característica clínica de la COVID-19, aún no se ha examinado su papel como marcador de gravedad.

Yao, et al. (2020) estudiaron la correlación entre la elevación del dímero D y la saturación de oxígeno, apoyándose de la tomografía computarizada de tórax, para evidenciar las áreas pulmonares más afectadas. De acuerdo con sus resultados, la mortalidad intrahospitalaria también se asoció con un incremento de las cifras de dímero $D$, lo que apoya que este puede utilizarse como un biomarcador clínico en SIRA ocasionado por el SARS-CoV-2.

Tang, et al. (2020) reportaron que en un análisis de 183 enfermos los valores de dímero $D$ fueron significativamente más altos en aquellos pacientes que fallecieron en comparación con las cifras reportadas en aquellos que sobrevivieron y con la población total $(2,12 \mu \mathrm{g} / \mathrm{ml}$, rango: $0.77-5.27 \mu \mathrm{g} / \mathrm{ml}$ vs. $0.66 \mu \mathrm{g} / \mathrm{ml}$, rango: $0.38-1.50 \mu \mathrm{g} / \mathrm{ml}$ y $0.61 \mu \mathrm{g} / \mathrm{ml}$, rango: $0.35-1.29 \mu \mathrm{g} / \mathrm{ml}$, respectivamente; $p<0.001)$. En nuestra población la mortalidad fue significativamente mayor en pacientes con dímero $D \geq 1,500$, con un $97.3 \%$ de mortalidad en este grupo comparada con un $76 \%$ en el grupo con dímero $D<1,499(p=0.003)$.

De acuerdo con lo reportado por Wu, et al. (2020), la asociación de SIRA con alteración en la coagulación, específicamente con la elevación del dímero $D$, fue significativamente más alta en comparación con los pacientes sin SDRA (diferencia: $0.52 \mu \mathrm{g} / \mathrm{ml}$; IC 95\%: $0.21-0.94 \mu \mathrm{g} / \mathrm{ml} ; \mathrm{p}<0.001$ ). En nuestra población se evidenció que en los pacientes con dímero $D \geq 1,500$ fue más frecuente el SIRA severo (76 vs. $56 \% ; p=0.033$ ).

\section{Conclusión}

Valores de dímero $D$ superiores a 1,500 parecen tener un rol predictor en la gravedad del SIRA, la necesidad de ventilación mecánica invasiva y el riesgo de mortalidad en pacientes con infección por SARSCoV-2. Se considera que incluso podría representar una herramienta útil para instaurar un tratamiento más agresivo a aquellos pacientes con cifras basales incrementadas y para guiar el uso de la anticoagulación profiláctica o terapéutica, y con ello buscar disminuir la tasa de mortalidad y las complicaciones tromboembólicas asociadas con el padecimiento.

\section{Financiamiento}

La presente investigación no ha recibido ninguna beca específica de agencias de los sectores públicos, comercial o sin ánimo de lucro.

\section{Conflicto de intereses}

Los autores declaran no tener conflicto de intereses.

\section{Responsabilidades éticas}

Protección de personas y animales. Los autores declaran que para esta investigación no se han realizado experimentos en seres humanos ni en animales.

Confidencialidad de los datos. Los autores declaran que han seguido los protocolos de su centro de trabajo sobre la publicación de datos de pacientes.

Derecho a la privacidad y consentimiento informado. Los autores han obtenido el consentimiento informado de los pacientes y/o sujetos referidos en el 


\section{artículo. Este documento obra en poder del autor de correspondencia.}

\section{Bibliografía}

1. Guan W-J, Ni Z-Y, Hu Y, Liang W-H, Ou C-Q, He J-X, et al. Clinical characteristics of coronavirus disease 2019 in China. N Engl J Med. 2020;382(18):1708-20.

2. Zhou F, Yu T, Du R, Fan G, Liu Y, Liu Z, et al. Clinical course and risk factors for mortality of adult inpatients with COVID-19 in Wuhan, China: a retrospective cohort study. Lancet. 2020;395(10229):1054-62.

3. Berlin DA, Gulick RM, Martinez FJ. Severe covid-19. N Engl J Med. 2020;383(25):2451-60.

4. Gibson PG, Qin L, Puah SH. COVID-19 acute respiratory distress syndrome (ARDS): clinical features and differences from typical pre-CO VID-19 ARDS. Med J Aust. 2020;213(2):54-56.e1.

5. Chu H, Zhou J, Wong BH-Y, Li C, Chan JF-W, Cheng Z-S, et al. Middle East respiratory syndrome Coronavirus efficiently infects human primary $T$ lymphocytes and activates the extrinsic and intrinsic apoptosis pathways. J Infect Dis. 2016;213(6):904-14

6. Bikdeli B, Madhavan MV, Jimenez D, Chuich T, Dreyfus I, Driggin E, et al. COVID-19 and thrombotic or thromboembolic disease: Implications for prevention, antithrombotic therapy, and follow-up: JACC state-of-theart review. J Am Coll Cardiol. 2020;75(23):2950-73.
7. Ferrandis R, Llau JV, Quintana M, Sierra P, Hidalgo F, Cassinello C et al. COVID-19: opening a new paradigm in thromboprophylaxis for critically ill patients? Crit Care. 2020;24(1):332.

8. Páramo JA. Respuesta inflamatoria en relación con COVID-19 y otros fenotipos protrombóticos. Reumatol Clin. 2020 Jun 17;S1699258X(20)30143-1. doi: 10.1016/j.reuma.2020.06.004. Online ahead of print.

9. Tang N, Li D, Wang X, Sun Z. Abnormal coagulation parameters are associated with poor prognosis in patients with novel coronavirus pneumonia. J Thromb Haemost. 2020;18(4):844-7.

10. Iba T, Levy JH, Connors JM, Warkentin TE, Thachil J, Levi M. The unique characteristics of COVID-19 coagulopathy. Crit Care. 2020;24(1):360.

11. Marchandot B, Sattler L, Jesel L, Matsushita K, Schini-Kerth V, Grunebaum L, et al. COVID-19 related coagulopathy: A distinct entity? J Clin Med. 2020;9(6):1651.

12. Colling ME, Kanthi Y. COVID-19-associated coagulopathy: An exploration of mechanisms. Vasc Med. 2020;25(5):471-8.

13. Moreno G, Carbonell R, Bodí M, Rodríguez A. Revisión sistemática sobre la utilidad pronóstica del dímero-D, coagulación intravascular diseminada y tratamiento anticoagulante en pacientes graves con COVID-19. Med Intensiva. 2021;45(1):42-55.

14. Barnes GD, Burnett A, Allen A, Blumenstein M, Clark NP, Cuker A, et al. Thromboembolism and anticoagulant therapy during the COVID-19 pandemic: interim clinical guidance from the anticoagulation forum. J Thromb Thrombolysis. 2020;50(1):72-81. 